\title{
COMPRO: A Methodological Approach for Business Process Contextualisation
}

\author{
Jose Luis de la Vara ${ }^{1}$, Raian $\mathrm{Ali}^{2}$, Fabiano Dalpiaz ${ }^{2}$, Juan Sánchez ${ }^{1}$, \\ and Paolo Giorgini ${ }^{2}$ \\ ${ }^{1}$ Centro de Investigación en Métodos de Producción de Software \\ Universidad Politécnica de Valencia, Spain \\ \{jdelavara, jsanchez\}@pros.upv.es \\ ${ }^{2}$ Department of Information Engineering and Computer Science \\ University of Trento, Italy \\ \{raian.ali, fabiano.dalpiaz, paolo.giorgini\}@disi.unitn.it
}

\begin{abstract}
Context-awareness has emerged as a new perspective for business process modelling. Business processes are strongly influenced by context, the environment where they are executed, and thus context should not be ignored when modelling them. This calls for new approaches that facilitate contextualisation, i.e. identification and representation of the way context influences a business process. In addition, detailed methodological guidance for correct business process contextualisation should be provided. However, existing works on context-aware business process modelling do not deal with these challenges. This paper addresses them by presenting COMPRO, a methodological approach for business process contextualisation. Starting from an initial business process model, context is analysed in order to discover its relevant variations and specify their effect on a business process. Our approach helps process designers to adequately specify context variants and business process variants that accommodate them. Our ultimate goal is to guarantee the correct design of business processes that fit their context. In addition, we report initial results about COMPRO application and evaluation.
\end{abstract}

Keywords: business process modelling, context-awareness, business process contextualisation, correctness of business process models.

\section{Introduction}

The importance of business process modelling nowadays is undeniable. It plays a major role in many fields such as business process management and information system development both in industry and in academia [10]. Traditional approaches for business process modelling have not paid much attention to the dynamism of the environment in which a business process is executed. However, organizations and their software systems currently operate in an environment in which changes are usual and need to adapt their behaviour in order to adequately operate when changes occur. As a result, the research community has acknowledged the importance of considering 
flexibility and variability during business processes modelling [10, 21]. Since business processes are influenced by their environment, business process modelling inevitably has to consider such factor and its variable nature.

Context-awareness has emerged as a new perspective for business process modelling in order to meet these needs [19]. It has already been applied in softwarerelated fields such as human computer interaction [5] and pervasive computing [9], and it is expected to improve business process modelling by explicitly addressing fitness between business processes and their context. Context should be analysed when modelling a business process to identify its context variants (relevant states of the world in which the business process is executed) and how they influence the business process, and to determine how the business process has to adapt to them.

Although several works have contributed to the advance of context-aware business process modelling (e.g. [8, 19, 22]), research on this topic is still at an initial stage. An important unexplored challenge is provision of techniques for determination of the relevant context properties that influence a business process. Process designers need to understand business process context, reason about it, analyse context variations (changes) and discover the relevant context properties [7, 16]. These properties must be known in order to be able to check if a given context variant holds.

Creation of contextualised business process models, which explicitly depict and support a set of context variants, should also be facilitated. Methodology is a top issue for business process modelling [10], and mechanisms and guidance for business process contextualisation must be provided in order to help process designers to properly represent and support context variants in business process models.

Furthermore, several properties should hold in contextualised business process models to guarantee its quality [26]. For example, a contextualised business process model should be correct. Correctness properties and their verification are important concerns for the research community [11], and they should always be taken into account when modelling business processes. As explained below, adding the context dimension to a business process involves new threats to its correctness, and this fact must be addressed when defining a contextualisation approach.

This paper aims to advance in research on context-aware business process modelling by dealing with the aforementioned challenges. The objectives of the paper are to determine how business process context can be analysed, how it can influence business processes, how to create contextualised business process models, and how to guarantee their correctness.

We achieve these objectives by defining COMPRO (COntextualisation Method for business PROcesses), a methodological approach for business process contextualization. The approach adopts context analysis [1,2], a technique that aims to support reasoning about context and discovery of context properties, and adapts it for business process modelling. COMPRO provides mechanisms and detailed guidance that help process designers to reason about business process context and to model business processes that fit their context and are correct. Context properties and variants are analysed in order to determine how they influence a business process, to guarantee that a business process is properly executed in all its context variants, and to correctly model contextualised business processes.

In addition, we report on preliminary evaluation of COMPRO. The approach has been applied and initially evaluated in organizations of several sectors, and this 
evaluation has allowed us to get important feedback from industry, to identify benefits and limitations of the approach and to determine future research directions.

The paper is organised as follows. Section 2 reviews related work. Section 3 describes COMPRO. Section 4 presents its application and the lessons learnt from it. Finally, Section 5 summarises our conclusions and future work.

\section{Related Work}

The notion of context plays an important role in fields such as pragmatics, natural language semantics, linguistics, cognitive psychology and artificial intelligence [4]. For business processes, context can be defined as the set of environmental properties that have an impact on process design and/or execution [19], i.e. properties that can influence and change business process execution and that should be analysed when designing a business process.

We review related work to business process contextualisation according to three categories: context-aware workflows, principles for context-aware business process modelling and modelling of context effect on business processes.

Smanchat et al. [22] provide a survey of works about context-aware workflows. For example, they deal with context-aware exception handling, management of context-aware workflow systems and context-aware execution languages. These works focus on provision of technological solutions for context-aware execution of business processes, but do not provide guidance to analyse business process context and to contextualise business processes.

Works on principles for context-aware business process modelling have addressed context-awareness from a more general and conceptual point of view. These works have analysed the different kinds of business process contexts [20] and the importance of external factors for contextualisation [19], and have defined models for context discovery from existing process instances [7], frameworks for context variations [16] and context-aware process management cycles [17]. Explicitly or implicitly, they all have acknowledged the need of analysis of business process context. Their main weakness is that they are too general and abstract. More detailed and systematic guidance is necessary to facilitate application of their ideas for business process contextualisation from a methodological perspective.

Other works provide mechanisms for modelling of context effect on business processes. Some examples are labelling of transitions [3], variant-specific adaptations [8] and context sensitive regions [14]. Although these works can help process designers to model contextualised business processes, their guidance is insufficient. They explain how to specify context effect in a business process model, but do not explain how to analyse business process context and discover its effect. Furthermore, just [8] addresses correctness of contextualised business process models, but only for its mechanism for modelling of context effect instead of from a general point of view.

Finally, we have presented initial work on and ideas about business process contextualisation in [6]. Their application, evaluation, extension and improvement have resulted in COMPRO. 


\section{COMPRO Description}

The main objective of COMPRO is to provide mechanisms and detailed guidance for correct modelling of business processes that fit their context and thus are properly executed in all the context variants of their business environment. Fig. 1 outlines COMPRO.

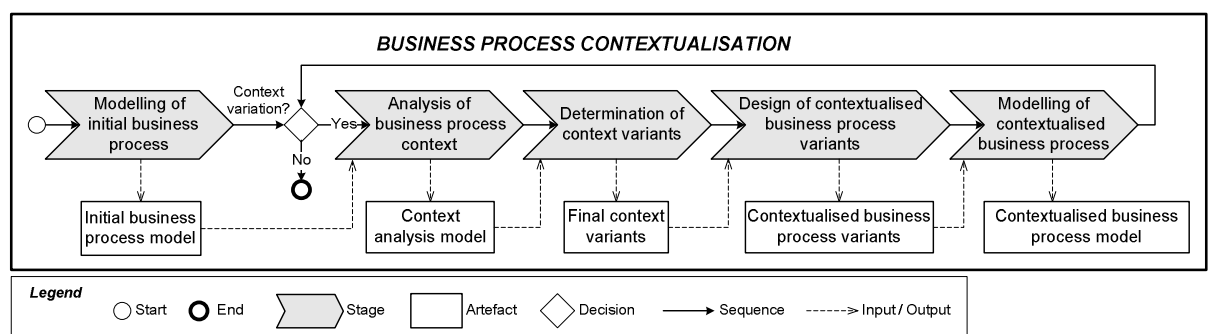

Fig. 1. Business process contextualisation through COMPRO

First, an initial version of a business process is modelled. Then, the following stages are iterated while relevant context variations are found and they are not represented and properly supported in the business process model. Relevant context variations can influence the business process and imply that business process execution has to change, i.e. tasks of the business process have to be either executed or not depending on context variations.

If a context variation is found, then business process context is analysed to find the context properties that allow business process participants to know if a given context variant holds. A context analysis model is created, and context variants of the business process have to be analysed in order to adequately specify them. Afterwards, contextualised business process variants are designed on the basis of the final context variants and their effect on the business process. Finally, a contextualised business process model is created from contextualised business process variants.

Our approach identifies relevant context variations and addresses flexibility and variability of business processes at design time [21]. As a result, it can increase process flexibility, decrease reaction time to context variations and improve risk management [19].

Throughout this section we use product promotion in a department store as a running example. Such scenario is an adaptation of a real business process of a Spanish company with which we applied COMPRO. Although it is relatively small, the example allows us to clearly show all the mechanisms and guidance of the approach.

After performing product promotion as a strategy for income increase for one year, company managers have realised that return on investment is negative and that product promotion resulted effective only in some sections. Company managers wonder why product sales did not sufficiently increase during promotion periods so that they covered the associated expenses, especially given the fact that product promotion works and is a common practice in competitors. 
Managers of the company need to make a decision about whether cancel the strategy or not. After discussing product promotion, they have decided to give it a new chance. They have also decided to analyse how products were promoted the previous year and how they should be promoted in future before launching any new product promotion. It is evident that the business process that was enacted failed, and a possible cause is that it did not properly fit the context in which it was executed. Analysis of the business process and of its context can help managers to find previous problems and to define new solutions so that product promotion does not fail again.

The following subsections describe each stage of COMPRO and present the mechanisms and guidance that are necessary to carry them out.

\subsection{Modelling of Initial Business Process}

In the first stage of COMPRO, an initial version of the business process that needs to fit its context is modelled. This model defines the context scope that has to be analysed, is the starting point for understanding of an organization and its context, and is used as basis for subsequent analysis of business process context.

The initial business process model can be created in different ways. For example, it could correspond to a reference model that will be adapted to the context of an organization, to a model that depicts the current behaviour of an organization and that needs to be contextualised for proper execution, or to a model that is created for a new scenario in an organization that was not previously needed. These situations may correspond to ERP implementation, business process reengineering and need of a new business process for new regulations compliance, respectively.

Fig. 2 shows the initial business process model for the running example and represents how product promotion has been executed. Although Fig. 2 has been modelled with BPMN (http://www.bpmn.org), COMPRO is not targeted to be only used with this notation.

When a product is promoted, section managers notify assistants of the promotion. Assistants have to find potential buyers for the product and to explain the promotion to them. If a customer is interested in and thus wants the promotion, then assistants give him the product to buy it. Assistants repeat this process until shift change.

The business process model does not reflect some relevant context variations and thus does not fit its context. For example, most of customers prefer not being addressed for product promotion unless they have addressed an assistant, especially when they are in a hurry. If an assistant addresses a customer that probably does not correspond to a potential buyer, then the customer can have a negative reaction about product promotion and the department store and may refuse product promotion in future. In addition, the assistant is missing the chance to address a real potential buyer, and addressing this customer may not be possible later. Consequently, assistants must be careful when looking for (finding) potential buyers. If context variations like these are disregarded, then product promotion may fail. They should be explicitly modelled so that assistants are aware of them and behave adequately.

Although all tasks (and thus the entire business process) of Fig. 2 could be a subject of contextualization, in the rest of Section 3 we focus on contextualization of the task "Find Potential Buyer". 


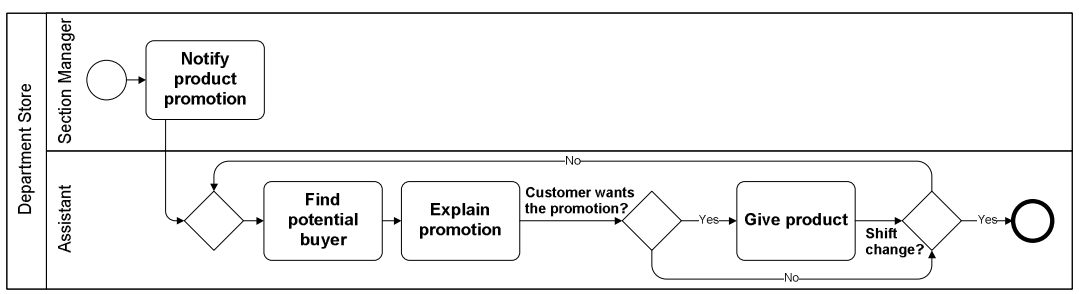

Fig. 2. Initial business process model

\subsection{Analysis of Business Process Context}

The second stage aims to understand context, to reason about it and to discover the context properties that influence a business process. For these purposes, context analysis [1, 2] has been adapted for analysis of business process context. This technique has been presented in the requirements engineering field, and is redefined from a business process perspective in COMPRO.

Context is specified as a world predicate formula. The EBNF of such formula is shown in Fig.3. World predicates are classified on the basis of their verifiability by a business process participant into two kinds: facts and statements.

Definition 1 (Fact) A world predicate $F$ is a fact for a business process participant $P$ iff $F$ can be verified by $P$.

A business process participant has a clear way to verify a fact. He possesses or can obtain the necessary data to objectively judge the truth value of a fact. For example, a world predicate such as "Customer asks for the product" can be verified by an assistant and its truth value will be the same for any person at a given moment.

Definition 2 (Statement) A world predicate $S$ is a statement for a business process participant $P$ iff $S$ cannot be verified by $P$.

Unlike facts, some world predicates are not objectively verifiable by a participant because of reasons such as lack of information and their subjective nature. For example, the world predicate "Customer may want to buy the product" is a statement. An assistant may guess that it is true, but he cannot guarantee it. However, the truth value of a statement can be assumed or supposed if there are evidences that support it.

Definition 3 (Support) A statement $S$ is supported by a formula of world predicates $F m$ iff $F m$ provides enough evidence of the possible truth value of $S$.

A statement can be supported both by facts and by other statements, which can also be supported by other formulas. The support relationship is transitive, thus statements can be iteratively refined to facts that provide enough evidence. For example, if a formula Fm1 supports a statement S1 and 'S1 AND Fm2' supports S2, then 'Fm1 AND Fm2' supports S2. 
Nonetheless, refinement of statements to formulas of facts is not always possible. For example, facts that support the statement "Customer wants to buy a present" may not be found. Analysis of business process context only deals with statements and thus contexts that can be refined to facts, i.e. they are judgeable.

Definition 4 (Judgeable Statement) A statement $S$ is judgeable iff there exist a formula of facts $F m$ that supports $S$.

Definition 5 (Judgeable Context) A context $C$ is judgeable iff $C$ can be specified as a formula of facts and judgeable statements $\mathrm{Fm}$.

Obtaining a judgeable context and discovering the formula that implies it can be considered the main purposes of analysis of business process context. The facts of the formula correspond to the context properties that characterise the context and its variants, and their truth values determine how a business process should be executed.

Facts that allow a context to be judgeable are discovered by analysing the formula that specifies the context. In this analysis, world predicates that correspond to statements have to be further analysed and refined until they are judgeable. If a nonjudgeable statement was found when analysing the formula of a context, then the statement would have to be discarded. This statement would not allow relevant context properties (facts) to be found, and the resulting context would not be judgeable.

A context analysis model is created to facilitate reasoning about business process context and discovery of the facts of the formula that implies it. An example is shown in Fig. 4, which corresponds to context analysis for the task "Find potential buyer" of Fig. 2.

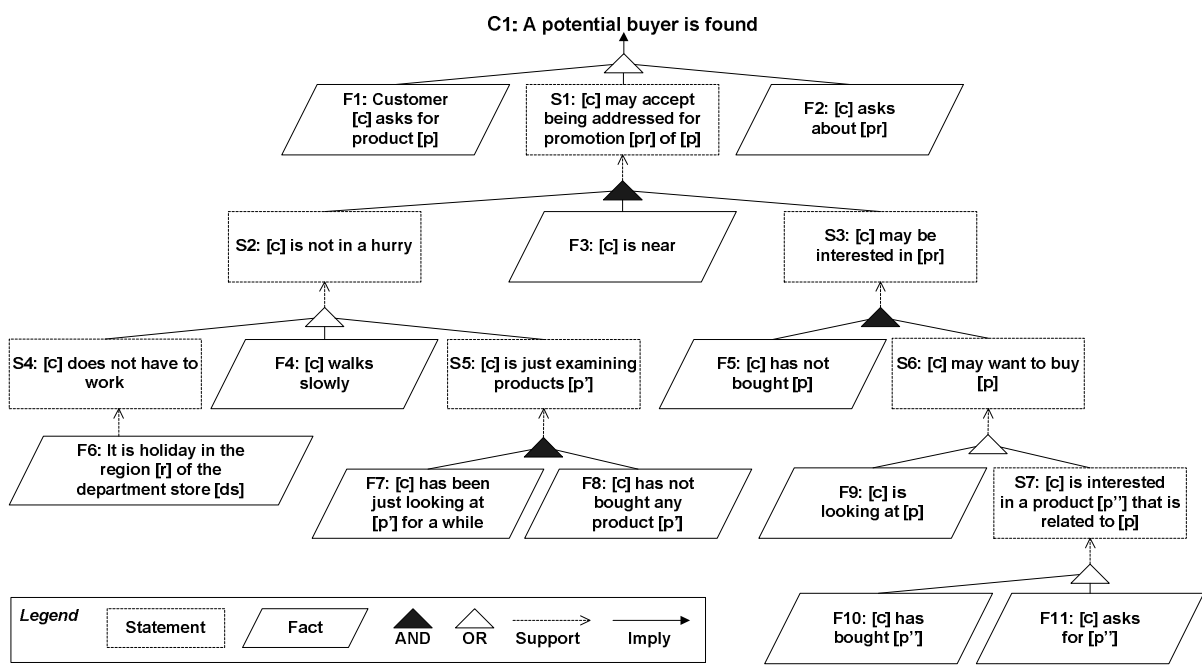

Fig. 4. Context analysis model 
A context analysis model is created by following these steps:

1. Determine the desired general context of the business process or fragment for which analysis is carried out.

2. Decompose the context to define the formula that specifies it.

3. Refine the statements of the formula until all of them are judgeable. If a statement is not judgeable, then it has to be removed from the model.

For Fig. 4, the desired context (C1) is "A potential buyer is found", the formula that implies it is 'F1 OR F2 OR (F3 AND (F4 OR F6 OR (F7 AND F8)) AND F5 AND (F9 OR F10 OR F11))', and all statements are judgeable. If the statement "Customer wants to buy a present" had refined S6, then it would have been removed.

Differently from other approaches for context modelling (see survey in [23]), context analysis does not provide an ontology or a modelling language for representing context, but modelling constructs to hierarchically analyse context. Nonetheless, a data conceptual model can be derived from facts of a context analysis model [1]. This model represents the information that has to be checked to verify the facts of the formula that implies a context.

\subsection{Determination of Context Variants}

Once a judgeable context has been found for a business process, context variants are determined in the third stage of COMPRO.

Definition 6 (Context variant) A context variant $C V$ is a set of facts whose conjunction implies a context $C$.

The main purposes of this stage are to adequately specify the (final) context variants in which the business process can be executed and that the context variants allow correct business process contextualisation and thus correct execution.

A context variant represents a state of the world in which a business process can be executed and (successfully) finished. For $\mathrm{C} 1$, a context variant corresponds to the set of facts ' $\{F 3$, F4, F5, F9 $\}$ ' (the formula 'F3 AND F4 AND F5 AND F9' implies the context). Fig. 5 (a) shows the eleven initial context variants for $\mathrm{C} 1$, which are derived from the formula that implies it.

As explained in the next subsection, contextualised business process variants have to be determined for each context variant of a business process and must allow the business process to be correct. Correctness of business processes is usually related to its soundness [26]. Soundness implies that, for a business process that is executed on the basis of a business process model, any business process instance can reach the final state, can only terminate in this state, and there are no dead transitions. If so, a business process is sound and all its business process executions will be correct.

Two situations can impede soundness of contextualised business process variants. The first one is that context variants contain conflicting facts, i.e. facts that cannot be true in a same business process instance. This situation is addressed in this stage. The second one is to follow a sequence of fact verifications that will not allow a business process instance to be finished, i.e. facts need to be verified in a given order so that verification of all of them is possible. This situation is addressed in the next stage. 


\begin{tabular}{|c|}
\hline Initial Context Variants \\
\hline$\{F 1\}$ \\
\hline$\{F 2\}$ \\
\hline$\{F 3$, F4, F5, F9 $\}$ \\
\hline$\{F 3, F 4, F 5, F 10\}$ \\
\hline$\{F 3, F 4, F 5, F 11\}$ \\
\hline$\{F 3, F 5, F 6, F 9\}$ \\
\hline$\{F 3, F 5, F 6, F 10\}$ \\
\hline$\{F 3, F 5, F 6, F 11\}$ \\
\hline$\{F 3, F 5, F 7, F 8, F 9\}$ \\
\hline$\{F 3, F 5, F 7, F 8, F 10\}$ \\
\hline$\{F 3, F 5, F 7, F 8, F 11\}$ \\
\hline
\end{tabular}

(a)

\begin{tabular}{|c|}
\hline Final Context Variants \\
\hline CV1: $\{\mathrm{F} 1\}$ \\
\hline CV2: $\{$ F2 $\}$ \\
\hline CV3: $\{\mathrm{F3}, \mathrm{F} 4, \mathrm{~F} 5, \mathrm{~F} 9\}$ \\
\hline CV4: $\{\mathrm{F} 3, \mathrm{~F} 4, \mathrm{~F} 5, \mathrm{~F} 10\}$ \\
\hline CV5:\{F4, F5, F11 $\}$ \\
\hline CV6: $\{\mathrm{F} 6, \mathrm{~F} 3, \mathrm{~F} 5, \mathrm{~F} 9\}$ \\
\hline CV7: $\{\mathrm{F} 6, \mathrm{~F} 3, \mathrm{~F} 5, \mathrm{~F} 10\}$ \\
\hline CV8:\{F6, F11, F5 $\}$ \\
\hline CV9:\{F3, F7, F8, F9\} \\
\hline CV10: $\{\mathrm{F} 11, \mathrm{~F} 7, \mathrm{~F} 8\}$ \\
\hline
\end{tabular}

(b)

Fig. 5. Context variants

The first situation is avoided by analysing the context variants of a business process in order to detect conflicts between facts. For this purpose, a table is created to specify the relationships between facts. The table also aims to obtain context variants whose set of facts are the minimum ones, i.e. no more facts are necessary (and thus do not need to be verified) to imply a context.

A table of relationships between facts of the running example is shown in Table 1, in which the relevant relationships (they affect context variants) are in bold and italics. For each cell of the table, the relationship between two facts is specified as follows:

Given a pair of facts Fr (fact of a row) and Fe (fact of a column),

- ' $\mathrm{X}$ ': no context variant contains Fr and Fc together, thus analysis is not necessary (this relationship can be automatically detected from initial context variants).

- 'Ur': Fr is always true when Fc is true, thus Fr verification will be unnecessary when $\mathrm{Fc}$ is true.

- ' $U c$ ' (opposite to 'Ur'): Fc verification will be unnecessary when Fr is true.

- 'C': Fr and Fc are conflicting.

- ' - ': no relationship exists between Fr and Fc.

For the running example, no analysis is necessary between F1 and other facts, F3 verification will be unnecessary if F11 is true, F8 and F10 are conflicting, and no relationship exists between $\mathrm{F} 7$ and $\mathrm{F} 8$.

As a result of the specification of the relationships between facts, the set of initial context variants is refined by removing conflicting variants and unnecessary facts. Refinement can be automated from a set of initial context variants and a table of relationships between facts, and is based on these two rules:

1) If two facts are conflicting, then the context variants that contain both facts are removed.

2) If the truth value of fact implies that verification of another is unnecessary, then the latter fact is removed from the context variants to which the first one belongs.

For the running example, the initial context variant ' $\{\mathrm{F} 3, \mathrm{~F} 5, \mathrm{~F} 7, \mathrm{~F} 8, \mathrm{~F} 10\}$ ' is removed because F8 and F10 are conflicting, and F3 is removed from the initial context variant ' $\{\mathrm{F} 3, \mathrm{~F} 4, \mathrm{~F} 5, \mathrm{~F} 11\}$ ' because its verification is unnecessary when F11 is true. Fig. 5 (b) shows the final context variants. 
Table 1. Relationships between facts

\begin{tabular}{|c|c|c|c|c|c|c|c|c|c|c|}
\hline & $\mathrm{F} 11$ & F10 & F9 & F8 & F7 & F6 & F5 & $\mathrm{F} 4$ & F3 & $\mathrm{F} 2$ \\
\hline $\mathrm{F} 1$ & $X$ & $X$ & $X$ & $X$ & $X$ & $X$ & $X$ & $X$ & $X$ & $X$ \\
\hline F2 & $X$ & $X$ & $X$ & $X$ & $X$ & $X$ & $X$ & $X$ & $X$ & \\
\hline F3 & $U r$ & - & - & - & - & - & - & - & & \\
\hline F4 & - & - & - & $\bar{X}$ & $\bar{X}$ & $\bar{X}$ & - & & & \\
\hline F5 & - & - & - & $U r$ & - & - & & & & \\
\hline F6 & - & - & - & $X$ & $\bar{X}$ & & & & & \\
\hline F7 & - & - & - & - & & & & & & \\
\hline F8 & - & $C$ & - & & & & & & & \\
\hline F9 & $\bar{X}$ & $\bar{X}$ & & & & & & & & \\
\hline F10 & $\bar{X}$ & & & & & & & & & \\
\hline
\end{tabular}

\subsection{Design of Contextualised Business Process Variants}

Contextualised business process variants of a business process are designed from the set of final context variants in the fourth stage of COMPRO.

Definition 7 (Contextualised business process variant) A contextualised business process variant $C B P V$ is an ordered set of fact verifications and task executions that specifies a correct execution of a business process for a context variant $C V$.

A contextualised business process variant corresponds to a possible execution of a business process that fits business process context and can be finished successfully. In addition, contextualised business process variants must allow a business process to be sound.

More specifically, the second situation in which a contextualised business process is not correct (sequence of fact verifications, which has been described in the previous subsection) is addressed in this stage. This situation is avoided by analysing the final context variants of a business process in order to determine the order of fact verification. For this purpose, a new table is created to specify the precedence between fact verifications. The table also aims to obtain efficient contextualised business process variants, i.e. no unnecessary actions are executed in them.

A table of precedence between fact verifications for the running example is shown in Table 2, in which the relevant precedence that is discovered (it affects contextualised business process variants) is in bold and italics. For each cell of the table, the precedence between two facts is specified as follows:

Given a pair of facts Fr (fact of a row) and Fe (fact of a column),

- ' $\mathrm{X}$ ': no context variant contains Fr and Fc together, thus analysis is not necessary (this relationship can be automatically detected from final context variants).

- 'Pr': Fc verification is only possible if $\mathrm{Fr}$ is true, thus $\mathrm{Fr}$ verification will precede Fc verification.

- 'Pc' (opposite to 'Pr'): Fc verification will precede Fr verification.

- ' $\mathrm{Kr}$ ': Fr truth value will be known before Fc verification.

- ' $\mathrm{Kc}$ ' (opposite to ' $\mathrm{Kr}$ '): Fc truth value will be known before Fr verification.

- ' - ': no relationship exists between Fr and Fc. 
Table 2. Precedence between fact verifications

\begin{tabular}{|c|c|c|c|c|c|c|c|c|c|c|}
\hline & $\mathrm{F} 11$ & F10 & F9 & F8 & F7 & F6 & F5 & $\mathrm{F} 4$ & F3 & $\mathrm{F} 2$ \\
\hline F1 & $X$ & $X$ & $X$ & $X$ & $X$ & $X$ & $X$ & $X$ & $X$ & $X$ \\
\hline F2 & $X$ & $X$ & $X$ & $X$ & $X$ & $X$ & $X$ & $X$ & $X$ & \\
\hline F3 & $X$ & $P r$ & $P r$ & $P r$ & $P r$ & $K c$ & $P r$ & $P r$ & & \\
\hline F4 & - & - & - & $X$ & $X$ & $X$ & - & & & \\
\hline F5 & $P C$ & - & - & $X$ & - & $K c$ & & & & \\
\hline F6 & $K r$ & $K r$ & $K r$ & $\bar{X}$ & $X$ & & & & & \\
\hline F7 & - & - & - & - & & & & & & \\
\hline F8 & $P C$ & $\bar{X}$ & - & & & & & & & \\
\hline F9 & $X$ & $\bar{X}$ & & & & & & & & \\
\hline $\mathrm{F} 10$ & $\bar{X}$ & & & & & & & & & \\
\hline
\end{tabular}

For the running example, no analysis is necessary between F1 and other facts, F3 verification will precede F10 verification, F6 truth value will be known before F11 verification, and no relationship exists between F7 and F8.

As a result of specification of relationships between facts, the order in which facts have to be verified for a given final context variant is specified. Specification of sequences of fact verification can be automated from a set of final context variants and a table of precedence between fact verifications, and is based on these two rules:

1) If verifications of several facts succeed or the truth values of the facts are known after verification of another, then the succeeding facts are put in brackets.

2) If verification of a fact precedes or the truth value of the fact is known before verification of another (or of a set of facts), then and arrow (' $\rightarrow$ ') is introduced in the specification of the final context variant. The arrow precedes the first fact and succeeds the second one.

For the running example, the context variant ' $\{\mathrm{F} 3, \mathrm{~F} 4, \mathrm{~F} 5, \mathrm{~F} 9\}$ ' turns into ' $\{\mathrm{F} 3 \rightarrow$ (F4, F5, F9) $\}$ ' because F3 verification precedes F4, F5 and F9 verification. Fig. 6 shows the set of sequences of fact verifications.

\begin{tabular}{|c|c|c|}
\hline Final Context Variants & \multirow[t]{11}{*}{$\rightarrow$} & Sequences of Fact Verification \\
\hline CV1: $\{\mathrm{F} 1\}$ & & $\{\mathrm{F} 1\}$ \\
\hline CV2: $\{F 2\}$ & & $\{\mathrm{F} 2\}$ \\
\hline CV3: $\{F 3, F 4, F 5, F 9\}$ & & $\{\mathrm{F} 3 \rightarrow(\mathrm{F} 4, \mathrm{~F} 5, \mathrm{~F} 9)\}$ \\
\hline CV4: $\{F 3, F 4, F 5, F 10\}$ & & $\{\mathrm{F} 3 \rightarrow(\mathrm{F} 4, \mathrm{~F} 5, \mathrm{~F} 10)\}$ \\
\hline CV5:\{F4, F5, F11 $\}$ & & $\{\mathrm{F} 4, \mathrm{~F} 11 \rightarrow \mathrm{F} 5\}$ \\
\hline CV6: $\{F 6, F 3, F 5, F 9\}$ & & $\{\mathrm{F} 6 \rightarrow \mathrm{F} 3 \rightarrow(\mathrm{F} 5, \mathrm{~F} 9)\}$ \\
\hline CV7: $\{F 6, F 3, F 5, F 10\}$ & & $\{\mathrm{F} 6 \rightarrow \mathrm{F} 3 \rightarrow(\mathrm{F} 5, \mathrm{~F} 10)\}$ \\
\hline CV8:\{F6, F11, F5\} & & $\{\mathrm{F} 6 \rightarrow \mathrm{F} 11 \rightarrow \mathrm{F} 5\}$ \\
\hline CV9:\{F3, F7, F8, F9\} & & $\{\mathrm{F} 3 \rightarrow(\mathrm{F} 7, \mathrm{~F} 8, \mathrm{~F} 9)\}$ \\
\hline CV10: $\{F 11, F 7, F 8\}$ & & $\{\mathrm{F} 7, \mathrm{~F} 11 \rightarrow \mathrm{F} 8\}$ \\
\hline
\end{tabular}

Fig. 6. Sequences of fact verification

The next step for design of contextualised business process variants is to determine the tasks that will be part of them. The tasks can correspond to three types: 1) tasks of the initial business process model; 2) tasks that are defined from refinement of the tasks of the initial business process model, and; 3) tasks that make facts true.

The first type corresponds to those tasks whose execution will not be influenced by context. The task "Give product" of Fig. 2 is of this type. The second type corresponds to tasks that were not initially modelled but that are considered necessary 
for proper execution of a business process and whose execution depends on context. For the running example, a task of this type is "Address customer", which refines "Find potential buyer". The third type corresponds to tasks that make facts true. If a task of this type is executed when a given fact is false, then the fact turns into true. These facts are called manageable.

Definition 8 (Manageable fact) A fact $F$ is manageable iff execution of a task $T$ makes $F$ true.

For the running example, F3 ("[c] is near") is manageable. If this fact is false, then an assistant can approach a customer (execute the task "Approach customer") so that the fact becomes true. Therefore, the task allows F3 to be manageable.

Once tasks are determined, a table is created to specify their relationships with the facts of the final context variants. An example is shown in Table 3 for the running example, in which the relevant relationships that are discovered (they affect contextualised business process variants) are in bold and italics. Tasks of the first type have not been included. For each cell of the table, the relationship between a fact and a task is specified as follows:

Given a fact $F$, a set of facts $\varphi$ and a task $T$ :

- 'M': T allows F to be manageable.

- ' $U$ ': $T$ execution will be unnecessary if $F$ is true.

- 'Sc': T cannot be executed unless $\mathrm{F}$ is true, thus $\mathrm{T}$ execution will succeed $\mathrm{F}$ verification.

- ' $\operatorname{ScX}$ ' (where ' $\mathrm{X}$ ' is a number that identifies different instances of this relationship): $T$ should only be executed if some fact of $\varphi$ is true, thus $T$ execution will succeed verification of the facts of $\varphi$.

- '-': no relationship exists between $\mathrm{F}$ and $\mathrm{T}$.

For the running example, T1 allows F3 to be manageable, T1 execution will be unnecessary if $\mathrm{F} 1$ is true, $\mathrm{T} 2$ execution will succeed $\mathrm{F} 3$ verification, $\mathrm{T} 2$ execution will succeed F4, F5, F6, F7, F8, F9, F9 and F11 verification, and no relationship exists between $\mathrm{T} 1$ and $\mathrm{F} 8$.

Table 3. Relationships between tasks and facts

\begin{tabular}{|l|c|c|c|c|c|c|c|c|c|c|c|}
\cline { 2 - 11 } \multicolumn{1}{c|}{} & F1 & F2 & F3 & F4 & F5 & F6 & F7 & F8 & F9 & F10 & F11 \\
\hline T1: Approach customer & $\boldsymbol{U}$ & $\mathbf{U}$ & $\boldsymbol{M}$ & - & - & - & - & - & - & - & $\boldsymbol{U}$ \\
\hline T2: Address customer & $\mathbf{U}$ & $\mathbf{U}$ & Sc & Sc1 & Sc1 & Sc1 & Sc1 & Sc1 & Sc1 & Sc1 & $\boldsymbol{U}$ \\
\hline
\end{tabular}

The final step of this stage is to specify the contextualised business process variants. It is carried by extending the sequences of fact verification of a business process with tasks. Specification of contextualised business process variants can be automated from a set of sequences of fact verifications and a table of relationships between tasks and facts, and is based on these three rules:

1) If execution of a task $T$ is unnecessary if a fact $F$ is true, then $T$ is not introduced in any sequence of fact verifications to which $\mathrm{F}$ belongs.

2) If verification of a fact $F$ or a set of facts $\varphi$ precedes execution of a task $T$, then $T$ and an arrow (' $\rightarrow$ ') are introduced in the sequences of fact verifications to which F or $\varphi$ belong. The arrow precedes T and succeeds $\mathrm{F}$ or $\varphi$. 
3) If a task $T$ allows a fact $F$ to be manageable, then a new contextualised business process variant is specified for each sequence of facts to which $\mathrm{F}$ belongs. The new contextualised business process variants are specified from the sequences of fact verifications by turning $F$ into ' $\neg F$ ' and introducing $T$ and an arrow (' $\rightarrow$ '). The arrow succeeds ' $\neg F$ ' and precedes $T$.

For the running example, $\mathrm{T} 1$ is not introduced in the sequence of fact verifications ' $\{\mathrm{F} 4, \mathrm{~F} 11 \rightarrow \mathrm{F} 5\}$ ' because its execution is unnecessary if F11 is true. The sequence of fact verifications ' $\{\mathrm{F} 3 \rightarrow(\mathrm{F} 4, \mathrm{~F} 5, \mathrm{~F} 9)\}$ ' produces the contextualised business process variants ' $\{\mathrm{F} 3 \rightarrow(\mathrm{F} 4, \mathrm{~F} 5, \mathrm{~F} 9) \rightarrow \mathrm{T} 2\}$ ' and ' $\{\neg \mathrm{F} 3 \rightarrow \mathrm{T} 1 \rightarrow(\mathrm{F} 4, \mathrm{~F} 5, \mathrm{~F} 9) \rightarrow \mathrm{T} 2\}$ ' because verification of F4, F5 and F9 precedes T2 execution and T1 allows F3 to be manageable. Fig. 7 shows all the contextualised business process variants.

\begin{tabular}{|c|c|}
\hline CBPV1: $\{F 1\}$ & CBPV8: $\{\mathrm{F} 6 \rightarrow \neg \mathrm{F} 3 \rightarrow \mathrm{T} 1 \rightarrow(\mathrm{F} 5, \mathrm{~F} 9) \rightarrow \mathrm{T} 2\}$ \\
\hline CBPV2: $\{F 2\}$ & CBPV9: $\{\mathrm{F} 6 \rightarrow \mathrm{F} 3 \rightarrow(\mathrm{F} 5, \mathrm{~F} 10) \rightarrow \mathrm{T} 2\}$ \\
\hline CBPV3: $\{\mathrm{F} 3 \rightarrow(\mathrm{F} 4, \mathrm{~F} 5, \mathrm{~F} 9) \rightarrow \mathrm{T} 2\}$ & CBPV10: $\{\mathrm{F} 6 \rightarrow \neg \mathrm{F} 3 \rightarrow \mathrm{T} 1 \rightarrow(\mathrm{F} 5, \mathrm{~F} 10) \rightarrow \mathrm{T} 2\}$ \\
\hline CBPV4: $\{\neg \mathrm{F3} \rightarrow \mathrm{T} 1 \rightarrow(\mathrm{F} 4, \mathrm{~F} 5, \mathrm{~F} 9) \rightarrow \mathrm{T} 2\}$ & CBPV11: $\{\mathrm{F} 4, \mathrm{~F} 11 \rightarrow \mathrm{F} 5\}$ \\
\hline CBPV5: $\{\mathrm{F} 3 \rightarrow(\mathrm{F} 4, \mathrm{~F} 5, \mathrm{~F} 10) \rightarrow \mathrm{T} 2\}$ & CBPV12: $\{F 6 \rightarrow F 11 \rightarrow F 5\}$ \\
\hline CBPV6: $\{\neg F 3 \rightarrow T 1 \rightarrow(F 4, F 5, F 10) \rightarrow T 2\}$ & CBPV13: $\{F 3 \rightarrow(F 7, F 8, F 9) \rightarrow T 2\}$ \\
\hline CBPVV: $\{$ F6 $\rightarrow$ F3 $\rightarrow(F 5, F 9) \rightarrow$ T2 $\}$ & CBPV14: $\{\neg F 3 \rightarrow T 1 \rightarrow(F 7, F 8, F 9) \rightarrow T 2\}$ \\
\hline \multicolumn{2}{|c|}{ CBPV15: $\{F 7, F 11 \rightarrow F 8\}$} \\
\hline
\end{tabular}

Fig. 7. Contextualised business process variants

\subsection{Modelling of Contextualised Business Process}

The purpose of the last stage is to correctly model a contextualised business process so that it supports and can be properly executed in all its context variants. Since the procedure to specify contextualised business process variants guarantees that they fit the analysed context and are correct, the contextualised business process model that is obtained also fits its context and is correct.

The contextualised business process model is created on the basis of the fact verification and task execution sequences of the contextualised business process variants. First, the contextualised business process variants are graphically represented (e.g. CBPV1 to 4 in Fig. 8). BPMN has been extended by labelling sequence flows for specification of conditions (formulas) that have to hold so that a sequence flow is executed. Fact and formula verification is represented by means of gateways (exclusive decisions), and tasks by means of BPMN tasks.
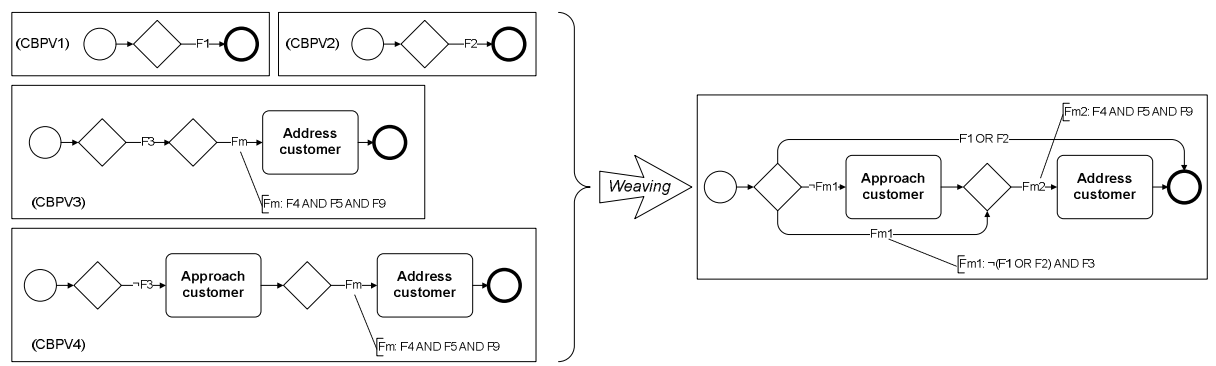

Fig. 8. Weaving of contextualised business process variants 
Second, the contextualised business process model is obtained by weaving together the graphical representations of the contextualised business process variants. An example of weaving is shown in Fig. 8, and Fig. 9 shows the result of contextualisation of the task "Find potential buyer" for the running example. The task has turned into a looping sub-process and has been divided into two sub-processes and four possible (groups of) paths execution: 'F1 OR F2', 'Fm1', ' $\neg$ Fm AND $\neg$ F11' and ' $\neg$ Fm1 AND F11'. The sub-processes include the tasks "Approach customer" and "Address customer". "Find potential customer" has to be executed until $\mathrm{C} 1$ is true.

Some parts of a contextualised business process model can be automatically derived from contextualised business process variants (e.g. modelling of a sequence of gateways and tasks), but human intervention and decisions may also be necessary (e.g. looping and sub-process modelling). This issue has to be further studied, and use of existing techniques for management of business process variants [12] have to be analysed (e.g. for their weaving). We also assume that soundness of a contextualised business process model still holds after human intervention.

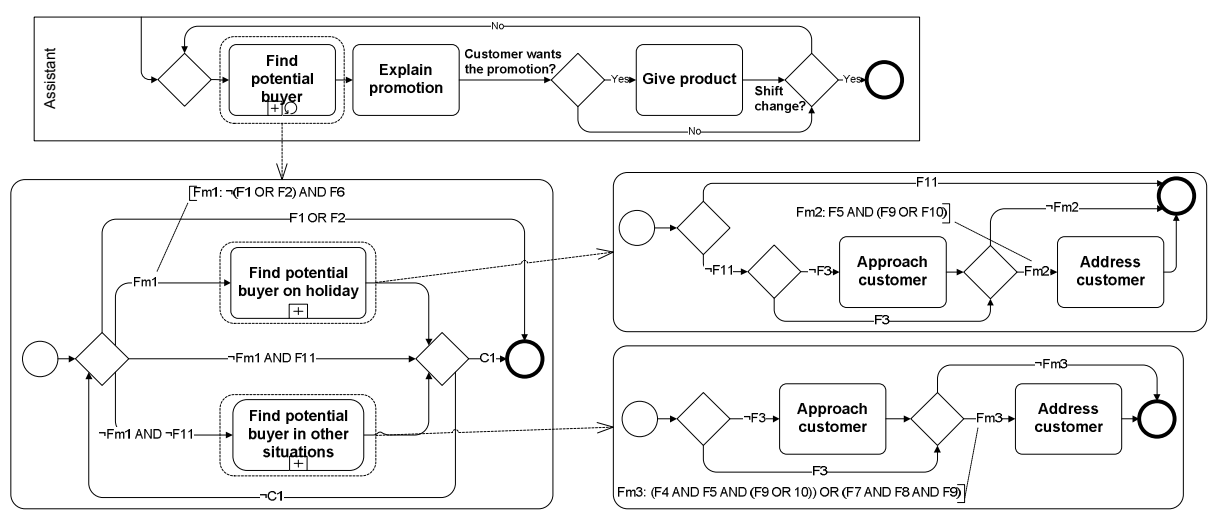

Fig. 9. Contextualised business process model

\section{COMPRO Application and Lessons Learnt}

This section presents and discusses COMPRO application (and thus its current evaluation), the considerations that arose from our experience and the lessons learnt.

By following an action research strategy [18], COMPRO has been applied and evaluated with business processes and stakeholders (managers, decision makers on business process execution and process designers) from eight organizations* of different sectors (automobile manufacture, construction, food and goods distribution and sale, public transport and software development). As a result, the approach has been defined and refined iteratively from needs and improvements that have been identified in its application. In this sense, applying COMPRO in industry examples has allowed us to identify its benefits and limitations.

\footnotetext{
${ }^{*}$ Many details of the organizations and thus of COMPRO application and evaluation are not presented for confidentiality reasons
} 
For evaluation, first we explained context-awareness for business process modelling to stakeholders. Second, we discussed the influence of context in their business processes. Third, we presented COMPRO and applied it collaboratively on at least one business process of the organization. Finally, we discussed with stakeholders about the approach. Evaluation was qualitative and data were collected through semistructured interviews.

The evaluation showed that context can have a substantial influence on business processes. A clear example of context influence that we encountered concerns piece manufacture and delivery in a provider of the automotive sector. The company manufactures pieces for an important carmaker, and have to meet very strict requirements. For example, the carmaker's plants follow a just-in-time strategy, which implies that pieces are not stored and that providers have to deliver them the day in which they are going to be assembled. If a provider fails to deliver the pieces and thus a plant has to stop manufacture, then the provider has to pay for the expenses that are derived from the stop. These expenses are very high, and have led some providers to bankruptcy. As a result, company managers have to check and control several context properties (related to, for instance, providers, production, weather and transport) in order to adapt the business processes of the company. Otherwise, pieces may not be delivered timely.

The main results, feedback and lessons learnt through COMPRO application with stakeholders are the following ones.

1) Good support for specification of indicators and for decision making

In general, most of the stakeholders regarded COMPRO as a means for discovery and specification of indicators that determine how a business process should be executed. The approach can help an organization to make decisions on business processes execution.

\section{2) Help for discovery of new context variants}

Although most of the context properties (facts) that were specified in the context analysis models were already known by the stakeholders, new context variants (both facts and their combination) were discovered in all organizations.

\section{3) Better fit between business processes and context}

All stakeholders stated that COMPRO could help their organizations to discover context variants and fit their business process to context. Systematic analysis of business process context and subsequent modelling of contextualised business process was considered very effective: organizations usually define responses to context variations in an ad-hoc way and do not try to discover new context variations (they have not happened yet).

\section{4) New expected advantages from business process contextualisation}

Although many works on business process contextualisation regard it as a problem of performance improvement (e.g. [19]), many stakeholders regarded it as a problem of exception discovery and definition of responses to them. This means that performance improvement is not the only and main expected advantage from business process contextualisation.

\section{5) Limitations in creation of context analysis model}

Creating a context analysis model is a slightly subjective activity. Statements are refined until stakeholders state that facts have been found, thus a context analysis model is considered to be finished on the basis of stakeholders perception. 
Furthermore, it is not possible to guarantee that a unique context analysis model exists for a given business process. In this sense, it is also not possible to guarantee that a context analysis model is complete (i.e. no more facts exist). This decision is based on stakeholders' validation.

\section{6) Difficulty in modelling of contextualised business processes}

If contextualised business process variants are woven together into a single contextualised business process model, then the model may get too tangled. We have used BPMN and labelling of sequence flows as mechanisms for modelling of contextualised business processes, but other approaches and mechanisms may be used. For example, declarative approaches for business process modelling (e.g. [15]) and/or mechanisms of related work that addresses modelling of context effect. We plan to analyse them in future.

\section{7) Need of COMPRO automation}

One of the main concerns of stakeholders was the availability of tool support for the approach in order to automate and facilitate its application. This is an essential point for future work. Integration of tool support for COMPRO with existing tools for business process modelling and management must also be addressed.

\section{8) Other suggestions from stakeholders}

Some stakeholders made comments about possible extensions of and improvements on COMPRO. All of them are not presented for the sake of brevity. For example, they asked about the definition of procedures for mitigation of the impact of context variations (before a context variant holds) in addition to definition of responses, and about specification of happening probability of facts and context variants. They have to be addressed in future.

We consider that the results from evaluation have been positive. Evaluation has not only allowed us to obtain very valuable feedback about the expected benefits of COMPRO, but also to identify relevant future work from a industry perspective. Nonetheless, the approach has to be further evaluated. We plan to execute case studies to check how practitioners use COMPRO on their own and benefit from it.

Last but not least, we are aware that COMPRO application and evaluation has not been presented as formally, systematically and in depth as it should be. For example, the interview instrument is not presented and threats to validity are not discussed due to page limitations.

\section{Conclusions and Future Work}

Context-aware business process modelling is an emerging topic and different benefits are expected from it. Nonetheless, further research has to be carried out to facilitate business process contextualisation. Approaches that address analysis of business process context and provide mechanisms and guidance for correct modelling of contextualised business process are necessary.

This paper has presented COMPRO, a methodological approach that addresses these challenges. The approach helps process designers to analyse business process context, to discover its relevant properties and variants, to determine their effect on a business process and to correctly contextualise it. 
COMPRO adopts context analysis and adapts it from a business process perspective. The approach also provides mechanisms and detailed guidance for analysis of business process context, context variants and business process variants and for modelling of contextualised business processes. They facilitate discovery and adequate specification of context properties (facts) and context variants, as well as of the relationships between facts and between facts and tasks of a business process. These relationships determine and constrain business process execution. Furthermore, the mechanisms and guidance aim to guarantee that a contextualised business process fits its context, is properly executed in all its context variants and is sound.

COMPRO application and current evaluation has shown its benefits and limitations. Since it facilitates identification of context variants and definition of responses to them, practitioners consider the approach to be useful. Nonetheless, some issues need to be addressed in order to meet some industry needs and to improve support for business process contextualisation.

As future work, we have mentioned several points of COMPRO that need more study or that may be carried out differently (Sections 4.4 and 5). Further work is especially important to maximize the industrial acceptance of business process contextualisation. In addition, we want to study the use of business process mining techniques (e.g. [25]) at analysis of business process context stage, of workflow patterns [24] and best practices for business process reengineering [13] at design of contextualised business process variants stage, and of layers of business process context (immediate, internal, external and environmental layers) [19] throughout COMPRO.

Acknowledgements. This work has been developed with the support of the Spanish Government under the projects SESAMO TIN2007-62894 and HI2008-0190 and the program FPU AP2006-02324, partially funded by the EU Commission through the projects COMPAS, NESSOS and ANIKETOS, and co-financed by FEDER. The authors would also like to thank all the people that participated in COMPRO application, and Amit K. Chopra and Richard Bertnsson Svensson for their useful comments on the paper.

\section{References}

1. Ali, R., Dalpiaz, F., Giorgini, P.: A Goal Modelling Framework for Self-Contextualizable Software. In: Halpin, T., et al. (eds.) BPMDS 2009 and EMMSAD 2009, LNBIP 29, Springer, pp. 326-338 (2009)

2. Ali, R., Dalpiaz, F., Giorgini, P.: A Goal-based Framework for Contextual Requirements Modeling and Analysis. Requirements Engineering Journal (to appear) (2010)

3. Born, M., Kirchner, J., Mueller, J.P.: Context-driven Business Process Modeling. In: TCoB 2009 (2009)

4. Bouquet, P., et al.: Theories and uses of context in knowledge representation and reasoning. Journal of Pragmatics 35(3):455-484 (2003)

5. Calvary, G., et al.: A Unifying Reference Framework for multi-target user interfaces. Interacting with Computers 15(3):289-308 (2003) 
6. de la Vara, J.L., et al.: Business Process Contextualisation via Context Analysis. In: ER 2010 (accepted paper) (2010)

7. Ghattas, J., Soffer, P., Peleg, M.: A Formal Model for Process Context Learning. In: S. Rinderle-Ma, et al. (eds.) BPM 2009 Workshops, LNBIP 43. Springer, pp. 140-157 (2010)

8. Hallerbach, A., Bauer, T., Reichert, M.: Capturing Variability in Business Process Models: The Provop Approach. Journal of Software Maintenance and Evolution: Research and Practice (in press) (2010)

9. Henricksen, K., Indulska, J.: A software engineering framework for context-aware pervasive computing. In: PerCom 2004, pp. 74-86 (2004)

10. Indulska, M., et al.: Business Process Modeling: Current Issues and Future Challenges. In: van Eck, P., Gordjin, J., Wieringa, R. (eds.) CAiSE 2009, LNCS 5565. Springer, pp. 501514 (2009)

11. Indulska, M., et al.: Business Process Modeling: Perceived Benefits. In: Laender, A.H.F., et al. (eds.) ER 2009, LNCS 5829. Springer, pp. 458-471 (2009)

12. la Rosa, M., Dumas, M., ter Hofstede, A.H.M.: Modelling Business Process Variability for Design-Time Configuration. In: Cardoso, J., van der Aalst, W. (eds.) Handbook of Research on Business Process Modeling. IGI Global, pp. 204-228 (2009)

13. Mansar, S.L., Reijers, H.A.: Best practices in business process redesign: validation of a redesign framework. Computers in Industry 56(5): 457-471 (2005)

14. Moddaferi, S., et al.: A Methodology for Designing and Managing Context-Aware Workflows. In: Krogstie, J., Kautz, K., Allen, D. (eds) Mobile Information Systems II. Springer, pp. 91-106 (2005)

15. Pesic, M., et al.: Constraint-Based Workflow Models: Change Made Easy. In: Meersma, R., et al. (eds) OTM 2007, Part I, LNCS 4803. Springer, pp. 77-94 (2007)

16. Ploesser, K., et al.: Context Change Archetypes: Understanding the Impact of Context Change on Business Processes. In: ACIS 2009, pp. 225-234 (2009)

17. Ploesser, K., et al.: Learning from Context to Improve Business Process. BPTrends 2009(1): 1-9 (2009)

18. Robson, C.: Real World Research. Blackwell, Oxford (2002)

19. Rosemann, M., Recker, J., Flender, C.: Contextualisation of business processes. International Journal of Business Process Integration and Management 3(1):47-60 (2008)

20. Saidani, O., Nurcan S.: Towards Context Aware Business Process Modelling. In: BPMDS'07 (2007)

21. Schonenberg, H., et al.: Process Flexibility: a Survey of Contemporary Approaches. In: Dietz, J.L.G., et al. (eds.) CIAO! 2008 and EOMAS 2008, LNBIP 10, Springer, pp. 16-30 (2008)

22. Smanchat, S., Ling, S., Indrawan, M.: A Survey on Context-Aware Workflow Adaptations. In: MoMM2008, pp. 414-417 (2008)

23. Strang, T., Linnhoff-Poppien, C.: A Context Modeling Survey. In: Workshop on Advanced Context Modelling, Reasoning and Management (UbiComp 2004) (2004)

24. van der Aalst, W.M.P., et al.: Workflow Patterns. Distributed and Parallel Databases 14(1):5-51 (2003)

25. van der Aalst, W.M.P., et al.: Business process mining: An industrial application. Information Systems 32(5): 713-732 (2007)

26. Weske, M.: Business Process Management: Concepts, Languages, Architectures. Springer (2007) 